\section{UPDATED SURVIVAL OF PATIENTS WITH PREVIOUSLY TREATED METASTATIC UVEAL MELANOMA WHO RECEIVED TEBENTAFUSP}

${ }^{1}$ Joseph Sacco*, ${ }^{2}$ Richard Carvajal, ${ }^{3}$ Marcus Butler, ${ }^{4}$ Alexander Shoushtari, ${ }^{5}$ Jessica Hassel, ${ }^{6}$ Alexandra lkeguchi, ${ }^{7}$ Leonel Hernandez-Aya, ${ }^{8}$ Paul Nathan, ${ }^{9}$ Omid Hamid, ${ }^{10}$ Josep Piulats, ${ }^{11}$ Matthew Rioth, ${ }^{12}$ Douglas Johnson, ${ }^{13}$ Jason Luke, ${ }^{14}$ Enrique Espinosa, ${ }^{15}$ Serge Leyvraz, ${ }^{16}$ Chris Holland, ${ }^{16}$ Michelle McCully, ${ }^{16}$ Shaad Abdullah, ${ }^{17}$ Takami Sato. ${ }^{1}$ The Clatterbridge Cancer Centre, Wirral, UK; ${ }^{2}$ Columbia University Medical Center, New York, USA; ${ }^{3}$ Princess Margaret Cancer Centre, Toronto, Canada; ${ }^{4}$ Memorial Sloan-Kettering Hospital, New York, NY, USA; ${ }^{5}$ University Hospital Heidelberg, Heidelberg, Germany; ${ }^{6}$ Stephenson Cancer Center, University of Oklahoma, Oklahoma City, USA; 'Washington University School of Medicine, St. Louis, USA; ${ }^{8}$ Mount Vernon Hospital, Northwood, UK; ${ }^{9}$ The Angeles Clinic and Research Institute, Los Angeles, CA, USA; ${ }^{10}$ Institut Català d'Oncologia, Barcelona, Spain; ${ }^{11}$ University of Colorado Cancer Center, Colorado, USA; ${ }^{12}$ Vanderbilt-Ingram Cancer Center, Nashville, TN, USA; ${ }^{13}$ UPMC Hillman Cancer Center, Pittsburgh, PA, USA; ${ }^{14}$ Hospital Universitario La Paz, Madrid, Spain; ${ }^{15}$ Charité Comprehensive Cancer Center, Berlin, Germany; ${ }^{16}$ Immunocore, Rockville, USA; ${ }^{17}$ Sidney Kimmel Cancer Center, Philadelphia, PA, USA

Background Tebentafusp, a bispecific fusion protein consisting of an affinity-enhanced $\mathrm{T}$ cell receptor fused to an anti-CD3 effector that can redirect $\mathrm{T}$ cells to target gp100+ cells, has shown an overall survival benefit for patients with untreated metastatic uveal melanoma (mUM) in a Ph3 trial (NCT03070392). Metastatic uveal melanoma (mUM) is a historically treatment-refractory tumor with 1-year (yr), 2-yr and 3 -yr OS rates of $37 \%, 15 \%$ and 9\%, respectively, and median OS of 7.8 months in $2 \mathrm{~L}+$ patients. $^{1}$ In the primary analysis of the phase 2 IMCgp100-102 study (NCT02570308) enrolling patients with previously treated mUM, the 1-year overall survival (OS) rate was $62 \%$ with median OS of 16.8 months. ${ }^{2}$ We present updated OS and safety after 2-year follow-up.

Methods 127 HLA-A*02:01+ 2L+ mUM patients were dosed weekly with tebentafusp following intra-patient dose escalation: $20 \mathrm{mcg}$ dose $1,30 \mathrm{mcg}$ dose 2 and $68 \mathrm{mcg}$ dose $3+$. Primary objective was ORR and secondary objectives included safety, OS and PFS. Here we present the updated OS and safety (data cut-off 31 Mar 2021).

Results Median follow-up was 29.9 mos (range 1.8 - 59.9 mos). With extended follow-up, the 1-yr, 2-yr and 3-yr OS rates were $61 \%, 37 \%$ and 24\%, respectively (figure 1). Median OS remained unchanged at 16.8 mos (95\% CI, 12.8 - 22.5 mos). Mean and median duration of treatment were 9.5 mos and $5.6 \operatorname{mos}(0-47.4$ mos), respectively. As previously reported, most treatment-related AEs (TRAEs) occurred early on treatment. Beyond 6 months, no TRAE led to treatment discontinuation. No new safety signals, changes in the type or treatment-related deaths were reported. Beyond 12 months, there were a total of 7 Grade $(G) 3$ or 4 events in $3(7 \%)$ patients, all were temporally related to tumor progression and majority included lab abnormalities. Episodes of rash, a common tebentafusp-related AE early on-treatment, were infrequent after 6 months, with no Grade 3 or 4 events.

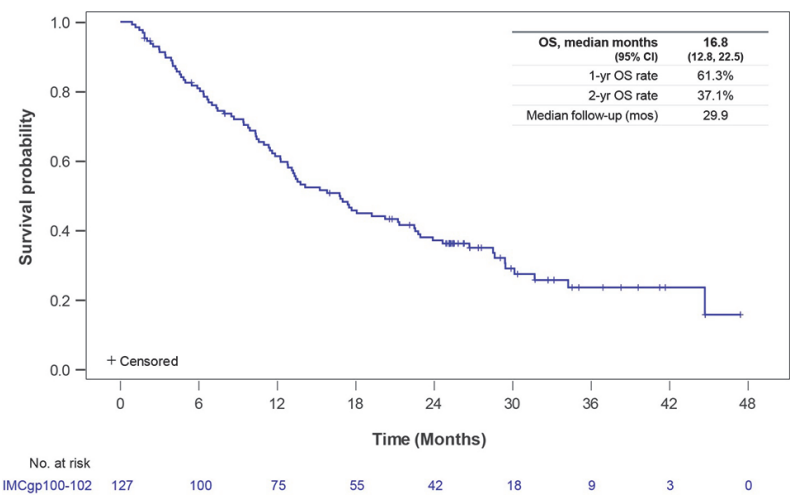

Abstract 538 Figure 1 Kaplan-Meier estimate of overall survival at 2-yr follow-up of IMCgp100-102

Conclusions This study provides the longest follow-up of OS and safety of a soluble TCR therapeutic to date. Tebentafusp continued to show promising survival for $2 \mathrm{~L}+\mathrm{mUM}$ patients with estimated 2-yr OS rate of 37\%. Tebentafusp's safety profile was as expected and consistent with primary analysis showing that most adverse events occur early on treatment with incidence and severity decreasing with prolonged exposure.

\section{Trial Registration NCT02570308}

\section{REFERENCES}

1. Rantala ES, Hernberg M, Kivela TT. Overall survival after treatment for metastatic uveal melanoma: a systematic review and meta-analysis. Melanoma Res 2019;29:561-568.

2. Sacco JJ, Carvajal R, Butler $\mathrm{MO}$, et al. A phase (ph) II, multi-center study of the safety and efficacy of tebentafusp (tebe) (IMCgp100) in patients (pts) with metastatic uveal melanoma (mUM). Ann Oncol 2020;31: S1442-S1143.

Ethics Approval The institutional review board or independent ethics committee at each center approved the trial. The trial was conducted in accordance with the Declaration of Helsinki and the International Conference on Harmonization Good Clinical Practice guidelines.

http://dx.doi.org/10.1136/jitc-2021-SITC2021.538 